J3eA, Journal sur l'enseignement des sciences et technologies de l'information et des systèmes, Volume 4, Hors-Série 1, 12 (2005)

DOI : http://dx.doi.org/10.1051/bib-j3ea:2005612

(C) EDP Sciences, 2005

\title{
Machines polyphasées : de la modélisation multimachine à la commande
}

E. Semail ${ }^{1}$, F. Meibody-Tabar ${ }^{2}$, M. F. Benkhoris ${ }^{3}$, H. Razik ${ }^{2}$, M. Pietrzak-David ${ }^{4}$, E. Monmasson ${ }^{5}$, A. Bouscayrol ${ }^{1}$, B. Davat ${ }^{2}$, Ph. Delarue ${ }^{1}$, B. de Fornel ${ }^{4}$, J. P. Hautier ${ }^{1}$, J. P. Louis ${ }^{5}$ et S. Piefederici ${ }^{2}$

${ }^{1}$ L2EP Lille, UPRES EA 2697, Université de Lille 1 (USTL)

F-59655 Villeneuve d'Ascq CEDEX, France

${ }^{2}$ GREEN, UPRES A 7037, ENSEM,

2 avenue de la Forêt de Haye, F-54600 Vandoeuvre les Nancy, France

${ }^{3}$ GE44, boulevard de l'Université

BP 406, F-44602 Saint-Nazaire, France

${ }^{4}$ LEEI, UMR 5828, ENSEEIHT

2 rue Camichel, F-31071 Toulouse CEDEX, France

${ }^{5}$ SATIE, UMR 8029, IUP GEII de Cergy

rue d'Eragny, Neuville sur Oise, F-95031 Cergy-Pontoise CEDEX, France

\author{
1,2,3,4,5 Projet SMM du GdR-ME2MS \\ Alain.Bouscayrol@univ-lille1.fr \\ URL : http: //www.univ-lillel.fr/l2ep/web-smm.htm \\ Eric.Semail@lille.ensam.fr \\ Farid.Meibody-Tabar@ensem.unancy.fr \\ mohamedfouad.benkhoris@polytech.univ-nantes.fr \\ Maria.David@leei.enseeiht.fr \\ Eric.Monmasson@iupge.ucergy.fr
}




\title{
Machines polyphasées: de la modélisation Multimachine à la commande
}

\author{
${ }^{1}$ L2EP Lille, UPRES EA 2697, Université de Lille 1 (USTL), 59655 Villeneuve d'Ascq cedex, Eric.Semail@ @ille.ensam.fr, \\ ${ }^{2}$ GREEN, UPRES A 7037, ENSEM, 2 avenue de la Forêt de Haye, 54600 Vandoeuvre les Nancy, Farid.Meibody-Tabar@ensem.u- \\ nancy.fr, \\ ${ }^{3}$ GE44, Boulevard de l'Université, BP 406, 44602 Saint-Nazaire, France, mohamedfouad.benkhoris@ polytech.univ-nantes.fr \\ ${ }^{4}$ LEEI, UMR 5828, ENSEEIHT, 2 rue Camichel, 31071 Toulouse cedex, Maria.David@leei.enseeiht.fr, \\ ${ }^{5}$ SATIE, UMR 8029, IUP GEII de Cergy, rue d'Eragny, Neuville sur Oise, 95031 Cergy-Pontoise Cedex, Eric.Monmasson@iupge.u- \\ cergy.fr, \\ 1,2,3,4,5 Projet SMM du GdR-ME²MS, Alain.Boucayrol@univ-lille1.fr, URL: http://www.univ-lille1.fr/l2ep/web-smm.htm
}

\author{
E. Semail ${ }^{1}$, F. Meibody-Tabar ${ }^{2}$, M. F. Benkhoris ${ }^{3}$, H. Razik ${ }^{2}$, M. Pietrzak-David ${ }^{4}$, E. Monmasson ${ }^{5}$,
} A. Bouscayrol ${ }^{1}$, B. Davat ${ }^{2}$, Ph. Delarue ${ }^{1}$, B. de Fornel ${ }^{4}$, J. P. Hautier ${ }^{1}$, J. P. Louis ${ }^{5}$, S. Piefederici ${ }^{2}$.

\begin{abstract}
Résumé-Un positionnement des systèmes polyphasés convertisseur-machine par rapport aux systèmes triphasés est proposé. Une représentation SMM (Systèmes Multimachine Multiconvertisseur) de ces systèmes polyphasés est introduite. Enfin, un cas particulier est étudié : une machine synchrone pentaphasée à aimant permanent alimentée par onduleur de tension.
\end{abstract}

\section{INTRODUCTION-PROBLEMATIQUE}

Le transport de l'énergie électrique par des réseaux triphasés a conduit par le passé au développement de convertisseurs électromécaniques triphasés. Ces derniers ont bénéficié de l'essor des interrupteurs de puissance et de celui des composants de commande type Digital Signal Processor (DSP). Les performances de ces classiques machines électriques triphasées associées à des onduleurs de tension s'en sont trouvées accrues notamment dans le domaine de la vitesse variable. Ce type de système est à présent largement disponible en standard au niveau industriel.

Néanmoins, le nombre réduit de phases peut être un handicap dans des domaines particuliers d'applications. Ainsi, des problèmes apparaissent tant au niveau de l'onduleur que de la machine lorsqu' on désire augmenter la puissance transmise. Les interrupteurs doivent en effet alors commuter des tensions et des courants d'amplitudes plus élevées, générant alors, lors des commutations, des perturbations électromagnétiques: l'augmentation de la tension induit des contraintes au niveau des diélectriques, l'augmentation du courant, des mises en parallèle d'interrupteurs.

Les onduleurs de tension multi-niveaux peuvent apporter une solution au prix d'un accroissement de la complexité de l'onduleur, particulièrement au delà de 3 niveaux de tension. Un fractionnement de la puissance par augmentation du nombre de phases de la machine est une autre solution [1]-[2] dans laquelle la complexité de conception et de commande s'équilibre mieux entre modulateur d'énergie et machine. Cette segmentation de la puissance répartit les contraintes sur plusieurs composants [3] de l'onduleur. Cela permet, entre autre, d'utiliser des composants de puissance fonctionnant avec une fréquence de commutation élevée, et ainsi, de réduire le taux harmonique, notamment celui du couple [3].

Dans le domaine particulier des réseaux embarqués tels l'automobile et l'avion, les choix des basses tensions et vitesses élevées impliquent au niveau des onduleurs, la commutation de forts courants avec des fréquences de commutation élevées. Un interrupteur est alors synthétisé à partir de nombreux composants mis en parallèle: on retrouve les problématiques de commutation synchronisée des composants, de surdimensionnement et de fiabilité associées.

Par ailleurs, un nombre élevé de phases confère intrinsèquement une meilleure fiabilité à l'ensemble convertisseur/machine. Des fonctionnements en modes de marches dégradées lors d'une mise en défaut d'un bobinage ou d'un composant sont possibles [4]-[5].

Des machines polyphasées ont ainsi été développées industriellement. La plus connue est sans doute la machine hexaphasée double étoile dont les deux étoiles sont déphasées électriquement de $30^{\circ}$. C'est initialement par commutateur de courant à thyristors que ces machines synchrones ont été alimentées [6]-[7]. Les commandes sont alors simples et ne nécessitent pas de traitement numérique de l'information: une commande analogique peut suffire. Dans ce type d'alimentations par créneaux de courant, l'emploi d'un nombre de phases élevé permet également de réduire les pulsations de couples tout en augmentant leur fréquence avec de ce fait un effet bénéfique sur les vibrations et le bruit. 
L'avènement des DSP permet à présent de s'intéresser à l'alimentation de ces machines par onduleur de tension commandé en Modulation de Largeur d'Impulsions. Il est alors possible de commander non seulement des machines synchrones [8]-[11] mais aussi des machines à induction [12]-[13]. Néanmoins, ce type d'alimentation nécessite une approche plus fine des machines polyphasées [3], [12]-[19]. En effet, dans le cas d'une alimentation par commutateur de courant d'une machine synchrone à q étoiles triphasées on peut considérer la machine polyphasée comme étant l'association sur un même arbre mécanique de $q$ machines triphasées, chacune étant associée à une des $q$ étoiles. Ceci est possible malgré les couplages magnétiques entre les différentes étoiles de par la particularité de l'alimentation par commutateur de courant : lorsqu'il y a commutation du courant dans une étoile, les courants sont constants dans les autres étoiles et n'interagissent donc pas via les mutuelles. Lors d'une alimentation en tension cette propriété n'est plus vérifiée. L'équivalence d'une machine polyphasée à un ensemble de machines plus simples n'est plus aussi triviale.

Depuis quelques années, les machines polyphasées alimentées par onduleur de tension ont fait l'objet de travaux au cas par cas, le nombre de phases étant alors une donnée et non un élément de choix. On trouve ainsi des études sur la commande de machine à réluctance variable [9],[25], de machines synchrones [8],[11],[26] et de machines à induction [1],[13],[29]. Quelques articles néanmoins comparent les qualités d'un type de machine en fonction du nombre de phases [20]-[22].

Le projet SMM (systèmes multimachines multiconvertisseurs) du GdR SDSE ${ }^{1}$ puis du GdR $\mathrm{ME}^{2} \mathrm{MS}^{2}$, a travaillé sur l'étude, la représentation synthétique et la commande de systèmes de conversion électromécanique composés de plusieurs machines et / ou convertisseurs statiques [34]-[37]. Dans la classification des SMM, les machines polyphasées correspondent à des systèmes à couplage magnétique. Elles sont ainsi perçues comme des machines "classiques" mettant en commun une carcasse magnétique afin de produire un couple commun à partir de plusieurs sources d'alimentation. Une étude préalable sur la généralisation du vecteur d'espace avait démontré qu'un système polyphasé pouvait se décomposer en plusieurs systèmes diphasés orthonormés pouvant être gérés de manières indépendantes [16]. Les travaux récents du projet SMM ont combiné les deux approches pour décomposer une machine polyphasée en machines fictives équivalentes possédant des propriétés intéressantes pour la commande [33]. Diverses études de machines pentaphasées ont été réalisées avec cette approche [10]-[11] et [30]-[32].

Cette communication présente le principe de décomposition de machines polyphasées en machines fictives monophasée et diphasées non couplées

\footnotetext{
1 Groupe de Recherche «Sûreté et disponibilité des Systèmes Electrique » du CNRS

${ }^{2}$ Groupe de Recherche «Maitrise de l'énergie électrique : du matériau au système » du CNRS
}

magnétiquement. Après la description de la méthodologie de décomposition, une machine synchrone pentaphasée est analysée avec son modèle de machines équivalentes.

\section{DeCOMPOSITION EN MaChines Fictives EQUIVALENTES}

\section{A. Formalisme SMM [34]}

Le formalisme SMM correspond à une représentation synthétique des conversions électromécaniques par des éléments interconnectés selon le principe de l'action et de la réaction. On distingue alors les éléments suivants (Fig. 1) :

- sources (générateur ou récepteur d'énergie, ovales verts)

- convertisseurs électriques (carrés orange),

- machines électriques (cercles orange),

- convertisseurs mécaniques (triangles orange).

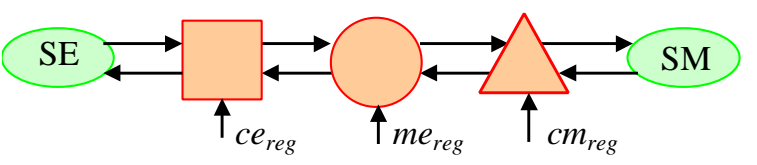

Fig. 1 : Chaîne élémentaire de conversion électromécanique

Des éléments de couplages sont introduits pour prendre en compte la distribution de l'énergie sur différentes chaînes de conversion (Fig. 2). Représentés par des pictogrammes imbriqués, ils sont considérés comme des entités "classiques" partageant une ressource physique commune et induisant une variable commune.

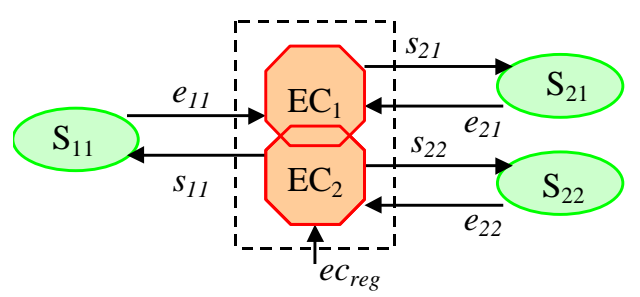

Fig. 2 : Exemple d'élément de couplage

\section{B. Généralisation du vecteur d'espace [16]}

Une machine polyphasée est composée de $n$ bobinages déphasés spatialement de $2 \pi / n$ et alimentés par des tensions déphasées temporellement de $2 \pi / n$. Ces machines sont caractérisées par un couplage magnétique entre phases.

Pour les machines triphasées, la transformation de Park ou la notion de vecteur d'espace (dimension 2) permet de trouver des bobinages fictifs équivalents qui simplifient l'étude. En effet, le couplage magnétique est réduit par des axes de travail orthogonaux. Cette opération correspond en fait à un changement de base.

La généralisation de la méthode du vecteur d'espace permet de définir un changement de base de dimension $n$, impliquant une simplification de l'étude de la machine par la diagonalisation de la matrice inductance. On montre alors que ce changement de base conduit à des sousespaces vectoriels orthogonaux de dimension 2 ou 1 . 
Chaque sous-espace peut ainsi être indépendant. La notion de machine fictive de dimension 1 ou 2 est alors introduite.

\section{Application aux machines polyphasées [19]}

La disparition du couplage magnétique induit par la transformation du vecteur d'espace généralisé, fait apparaître un couplage électrique et un couplage mécanique (Fig. 3).

Le couplage électrique correspond à la transformation mathématique qui lie les phases d'origine aux phases fictives, $v_{k}$ étant la tension aux bornes de la phase $n^{\circ} k$ de la machine. Quant au couplage mécanique, il est en fait relativement simple car il définit le couple total comme étant la somme des couples des machines fictives.

Ainsi, on transforme donc un couplage magnétique contraignant et difficile à gérer (cf. tous les travaux sur les commandes vectorielles) en couplages électriques et mécaniques simples. De plus, les machines fictives équivalentes sont de dimension 1 ou 2 . Or ce type de machine fictive est bien connu : bobinages équivalents dans le repère $(\mathrm{d}, \mathrm{q})$ de Park par exemple.

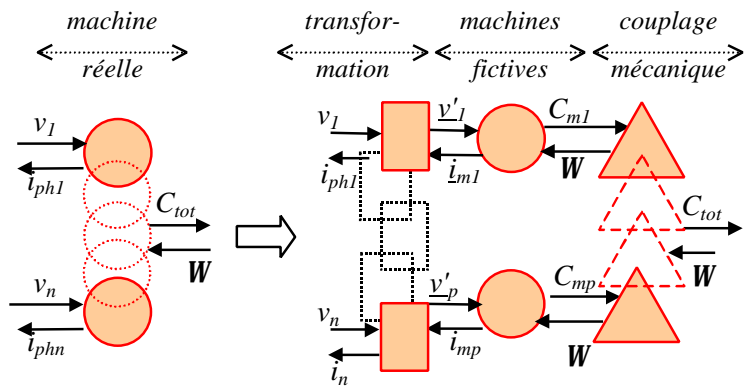

Fig. 3 : Couplages de la machine réelle et des machines fictives

\section{APPLICATION A UN CAS STANDARD}

\section{A. Structure étudiée}

Une machine synchrone pentaphasée est alimentée par 5 onduleurs monophasés (Fig. 4). Chaque phase est ainsi alimentée par son propre onduleur ce qui permet un fonctionnement en mode dégradé lors d'un défaut d'un composant de puissance ou d'un bobinage.

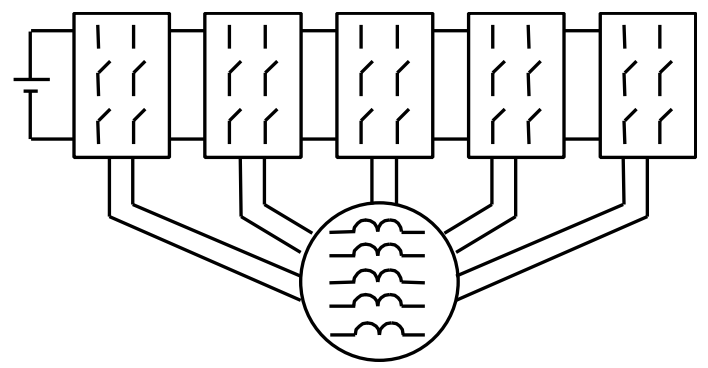

Fig. 4 : Onduleurs monophasés pour machine synchrone pentaphasée

Une alimentation de type MLI intersective classique fait apparaître de fortes ondulations de courants (Fig. 5), autour de la référence sinusoïdale demandée. Ces ondulations sont inattendues au vu du rapport faible entre la période de MLI et la constante de temps définie classiquement par $L_{c} / R$ (avec $R$ résistance d'une phase et $L_{c}$ inductance cyclique dans le cadre d'une approche au premier harmonique).

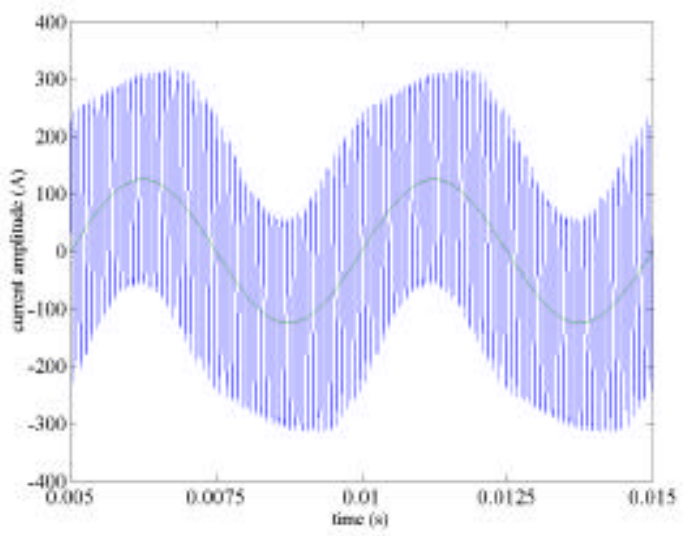

Fig. 5 : Courant dans une phase de la machine

\section{B. Représentation SMM}

La représentation SMM du système est composée des 5 onduleurs monophasés (carrés) et de la machine pentaphasée considérée comme 5 machines partageant la même carcasse (cercles imbriqués). Par souci de simplification, la source de tension continue est supposée idéale. Elle est alors représentée par 5 sources électriques qui fournissent la même tension $V_{D C}$ (Fig. 6) (règle de simplification des couplages [35].

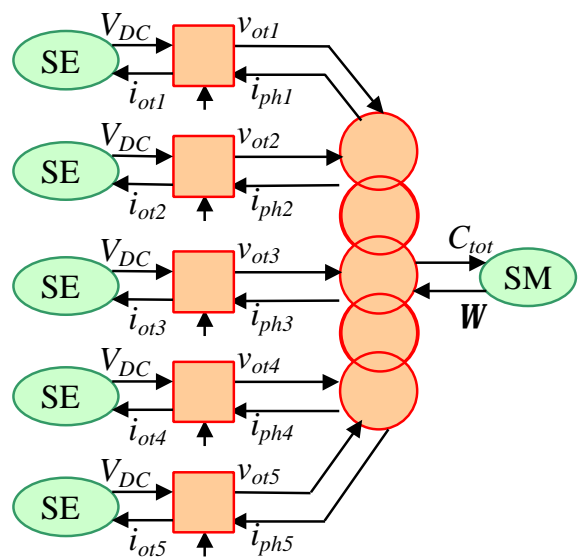

Fig. 6 : Description SMM du système étudié avec machine réelle

Les hypothèses suivantes sont adoptées pour modéliser la machine:

- toutes les phases sont identiques et régulièrement décalées par un angle $\alpha=2 \pi / 5$;

- pas d'effet de réluctance variable (pôles lisses);

- pas d'effet de saturation (circuit magnétique linéaire) Toutes les quantités concernant la phase $k$ sont écrites $x_{k}$. La machine pentaphasée est représentée schématiquement sur la Fig. 7. 


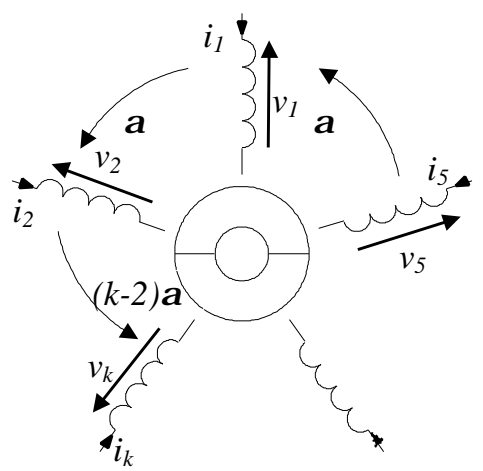

Fig. 7 : Présentation de machine synchrone de pentaphasée

L'analyse de la matrice des inductances propres, symétrique et circulante permet de mettre en évidence 3 valeurs propres $L_{c k}$, appelées inductances cycliques, et 3 espaces propres orthogonaux entre eux : deux plans et une droite.

On décompose de façon unique tout vecteur en une somme de 3 vecteurs appartenant chacun à l'un des 3 sous-espaces . A chaque plan on associe, un phaseur complexe, vecteur de dimension 2. A la droite, on associe un scalaire, vecteur de dimension 1. On obtient finalement les 3 équations suivantes faisant apparaître chacune une valeur propre $L_{c 0}$, $L_{c l}, L_{c 2}$ :

- $v_{0}=R i_{0}+L_{c 0} \frac{d i_{0}}{d t}+e_{0}$

- $\quad \underline{v}_{d q 1}=R \underline{i}_{d q 1}+L_{c 1} \frac{d}{d t} \underline{i}_{d q 1}+\underline{e}_{d q 1}$

- $\quad \underline{v}_{d q 2}=R \underline{i}_{d q 2}+L_{c 2} \frac{d}{d t} \underline{i}_{d q 2}+\underline{e}_{d q 2}$

Étant donné que les 3 sous-espaces propres sont orthogonaux, le bilan de puissance peut se décomposer en une somme de trois bilans élémentaires indépendants. Il est alors permis d'associer à chaque sous-espace propre une machine fictive participant à la création du couple de la machine réelle. En considérant que toutes les machines fictives tournent à la même vitesse $\Omega$, il vient que le couple total est simplement la somme des trois couples des machines fictives. Le couplage mécanique est donc très simple et on obtient alors la représentation proposée sur la Fig. 8 où le changement de base est représenté par un couplage électrique.

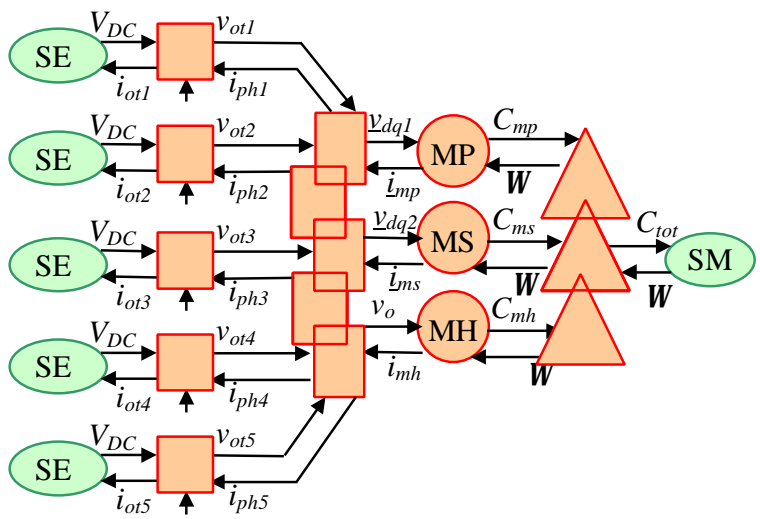

Fig. 8 : Description SMM du système étudié avec machines fictives

L'analyse de ces trois machines met en évidence, dans le cas d'une force électromotrice sinusoïdale, qu'une seule des 3 machines peut produire du couple. On appelle machine principale cette machine diphasée qui est liée à l'harmonique de rang 1 et dont l'inductance cyclique est notée $L_{c l}$.

Si par contre les forces électromotrices ne sont pas sinusoïdales alors chaque machine peut contribuer au couple, en fonction de l'amplitude de sa force électromotrice [11]. Ainsi, la deuxième machine diphasée pourra produire du couple principalement grâce aux harmoniques de rang 3 et 7 . Quant à la machine monophasée, la production de son couple pulsatoire est liée aux multiples de rang 5.

On a donc ainsi remplacé le couplage magnétique par un couplage électrique simple (matrice de transformation) et un couplage mécanique simple (association des couples). Cette représentation va être utilisée dans un premier temps pour l'analyse des résultats obtenus dans le cas de l'alimentation MLI intersective. On projette pour cela le vecteur courant dans les 3 sous espaces propres définis pour obtenir les courants des machines fictives (Fig. 9, Fig. 10, Fig. 11).

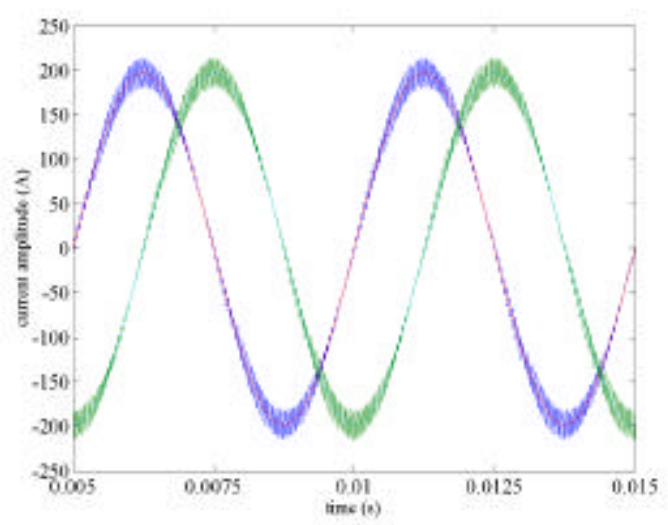

Fig. 9 : Courants dans machine principale, MLI intersective

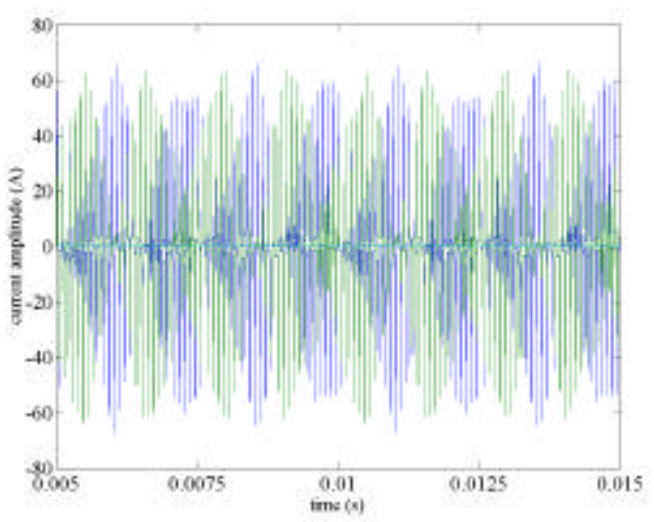

Fig. 10 : Courants dans machine secondaire, MLI intersective 


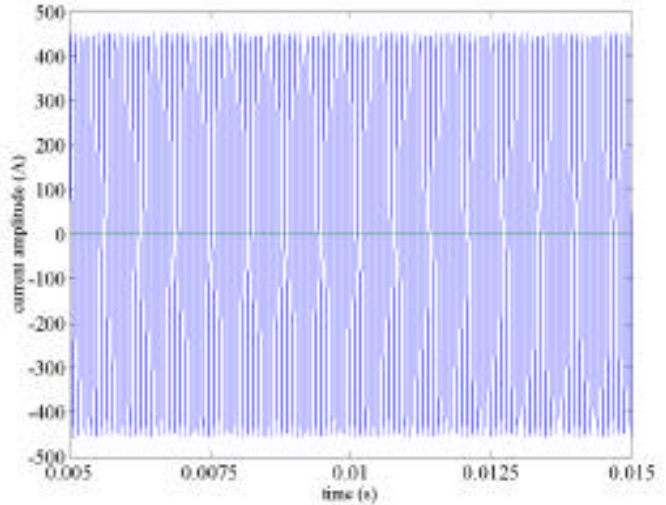

Fig. 11 : Courant dans la machine homopolaire, MLI intersective

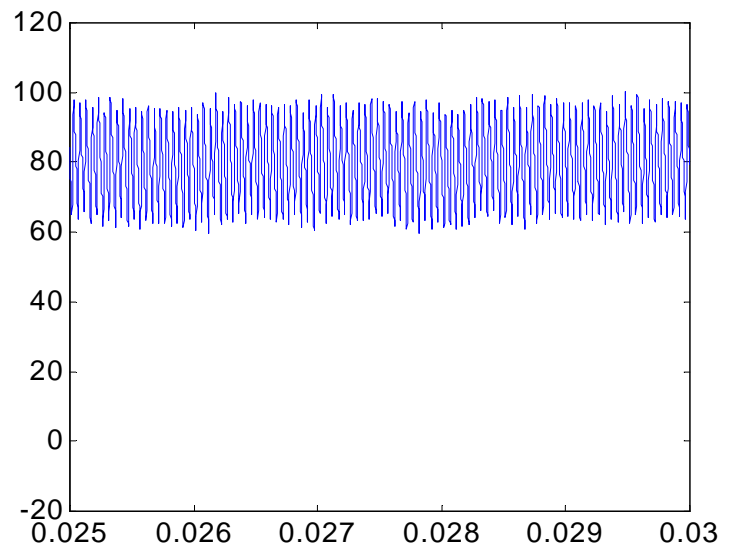

Fig. 12 : Couple électromagnétique de la machine.

Il apparaît alors que le taux d'ondulation des courants dans la machine principale est faible, conformément au rapport entre période de MLI et constante de temps $L_{c l} / R$. Par contre, il est manifeste que les constantes de temps des machines secondaire et homopolaire $L_{c 2} / R$ et $L_{0} / R$ sont plus faibles que celle de la machine principale. Même si ces dernières ne sont pas alimentées aux valeurs moyennes, elles le sont aux valeurs instantanées, des courants parasites s'y développant alors.

\section{Commande déduite}

La commande de la machine permet de contrôler le couple qu'elle fournit. C'est donc un couple $\mathrm{C}_{\text {tot-ref }}$ qui sera considéré. La présence du couplage mécanique met immédiatement en évidence alors la nécessité d'élaborer trois consignes de couple, une par machine fictive, à partir d'une seule consigne. La relation étant non bijective, il est nécessaire de définir des critères de répartition entre les différentes machines [36].

L'analyse des potentialités des machines secondaire et homopolaire met en évidence, dans la mesure où leurs forces électromotrices sont de faible amplitude par rapport à celle associée à la machine principale, que ces machines ne peuvent produire que peu de couple [33]. En revanche, leurs faibles impédances vis à vis de l'alimentation en tension est à l'origine de l'apparition de courants de forte amplitude, courants parasites dans la mesure où ils ne produisent pas de couple.

De cette représentation et analyse on peut proposer une commande des trois machines fictives [10]. La machine principale est commandée pour fournir à elle seule le couple total. Les autres machines sont commandées pour minimiser les courants parasites et donc les ondulations de courant.

Par conséquent une clé de répartition peut être la suivante :

- un couple de référence nul pour la machine secondaire et la machine homopolaire ;

- un couple de référence égal au couple total $C_{\text {tot-ref }}$ pour la machine principale ;

- une alimentation des machines secondaire et homopolaire de façon à réduire l'amplitude des courants parasites qui s'y développent.

Pour répondre à la dernière contrainte, il faut donner fournir une représentation des 5 onduleurs monophasés pour chaque machine. Il suffit pour cela de projeter les vecteurs tensions définis par les 32 combinaisons ${ }^{3}$ liées à l'état des interrupteurs.

Pour simplifier la mise en évidence de la commande, on prendra dans la suite le cas d'une machine à forces électromotrices sinusoïdales. Les forces électromotrices des machines secondaire et homopolaire sont alors nulles. Les équations en tension deviennent alors :

- $v_{0}=R i_{0}+L_{c 0} \frac{d}{d t} i_{0}$

- $\quad \underline{v}_{d q 1}=R \underline{i}_{d q 1}+L_{c 1} \frac{d}{d t} \underline{i}_{d q 1}+\underline{e}_{d q 1}$

- $\underline{v}_{d q 2}=R \underline{i}_{d q 2}+L_{c 2} \frac{d}{d t} \underline{i}_{d q 2}$

L'observation des courants dans les machines fictives (Fig. 13, Fig. 14) fait apparaître que l'ondulation est comparable dans la machine secondaire mais plus importante dans la machine principale. Par contre, l'observation des courants dans la machine met en évidence une réduction forte des ondulations, la machine homopolaire n'étant plus alimentée (Fig. 16 à comparer avec la Fig. 5). On a ainsi fortement amélioré la qualité des courants qui sont appelés par la machine permettant ainsi de ne pas surdimensionner les interrupteurs. Pour les couples, la fréquence des ondulations est multiple de la fréquence de la Modulation de Largeur d'Impulsions. Le filtrage par les charges mécaniques est donc aisé.

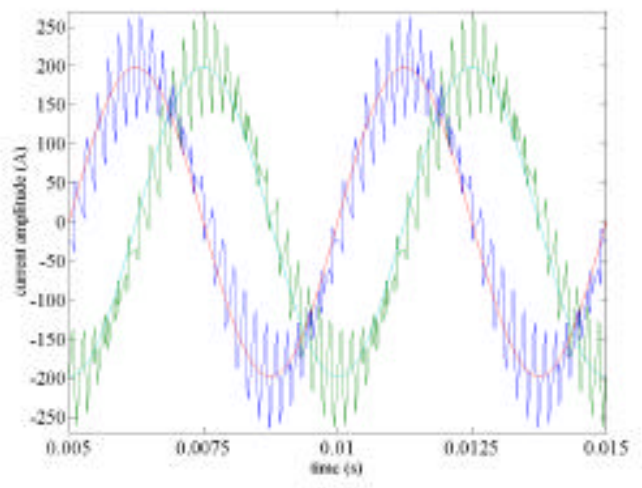

Fig. 13 : Courants dans machine principale, « commande $h=0$ »

\footnotetext{
$332=2^{5}$ On se limite dans un premier temps à une commande 2 niveaux : $+\mathrm{V}_{\mathrm{DC}},-\mathrm{V}_{\mathrm{DC}}$
} 


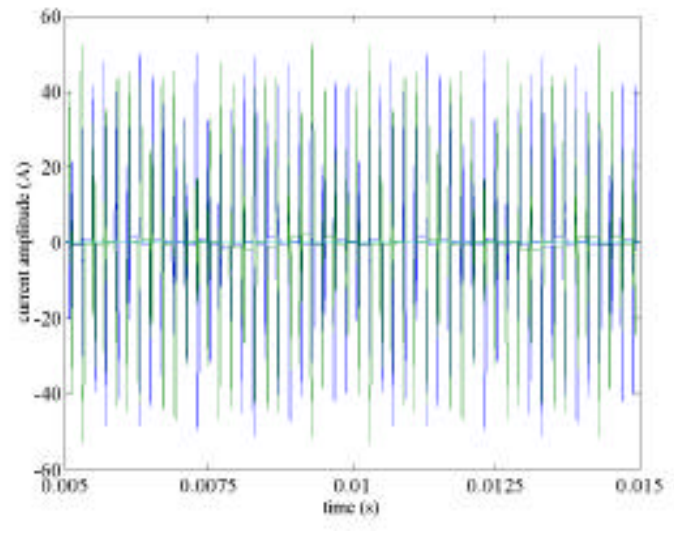

Fig. 14 : Courants dans machine secondaire, « commande $\mathrm{h}=0 »$.

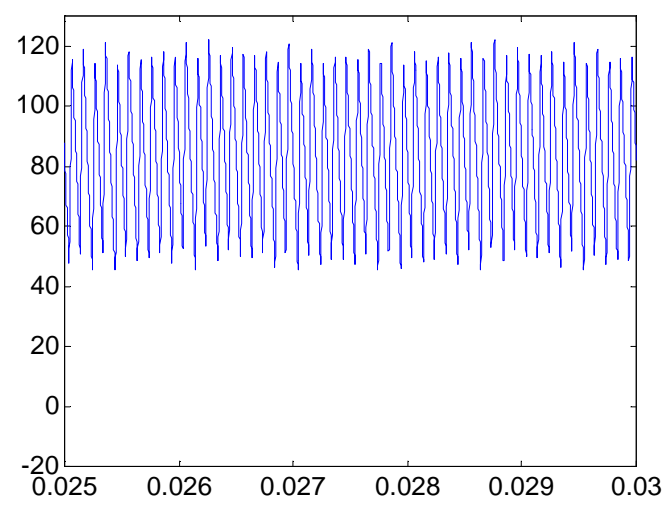

Fig. 15 : Couple électromagnétique de la machine, « commande h=0. »

\section{RÉFÉRENCES}

[1] E.A. Klingshirn, « High phase order induction motors _ Part I and II «IEEE Trans. Power Apparatus Syst., vol PAS -102, pp. 47-59, 1983.

[2] P. Letellier, «High Power Permanent magnet machines for electric propulsion drives » AES 2000 26/27 october 2000 Paris, France.

[3] N. Moubayed, F. Meibody-Tabar, B. Davat, "Alimentation par deux onduleurs de tension d'une machine synchrone double étoile", Revue Internationale de Génie Electrique, vol. 1, n 4, 1998, pp. 457-470.

[4] Norton P.T., Thompson "The naval electric ship of today and tomorrow", AES 2000, October 2000 Paris (France), pp. 80-86.

[5] T. Jahns, "Improved reliability in solid state ac drives by means of multiple independent phase-drive units", IEEE Trans. on Industry Applications, vol. 16, May-June 1980, pp 321-331.

[6] Bhatia R., Krattiger H., Bonanini A., Schafer D., Inge J.T., Sydnor G.H., "Adjustable Speed Drive With A Single 100-MW Synchronous Motor", ABB Review Issue, No6, 1998, pp.14-20.

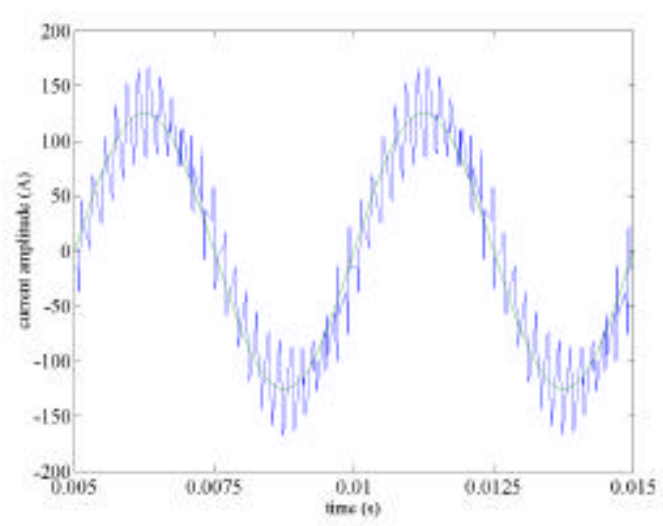

Fig. 16 : Courant de la phase réelle, « Commande $\mathrm{h}=0$ »

\section{CONCLUSION}

La combinaison des outils formalisme SMM et vecteur d'espace généralisé mène à la décomposition d'une machine polyphasée en machines fictives équivalentes. Cette représentation permet alors une analyse intéressante de ce type de machine. Elle conduit à des structures de commande originales et performantes.

Cette approche développée pour la machine pentaphasée peut bien sûr être étendue à d'autres machines [19]. Parmi les travaux déjà engagés dans le projet SMM, on peut citer les travaux sur les machines double étoile [18] et les machines double alimentation [38]. De plus cette méthode permet d'aborder facilement les marches en mode dégradé [33]. On peut penser que ces apports devraient permettre une utilisation croissante des machines polyphasées dans les applications industrielles et ce même en faible puissance. En effet, les possibilités de fonctionnement en marche dégradée offrent de réels intérêts pour des systèmes embarqués, en particulier pour des actionneurs du domaine aéronautique pour lesquels les contraintes de fiabilité sont élevées.

[7] Godfroi H., Bosc P., "Large variable speed drives using synchronous motors and frequency converters", Alstom Review $\mathrm{n}^{\circ} 6,1986$.

[8] S. Siala S., E. Guette, J. L. Pouliquen, "Multi-inverter PWM control: a new generation drives for cruise ship electric propulsion", European Power Electronics Conference (EPE'2003), September 2003, Toulouse (France), CD-ROM.

[9] Toliyat H.A., Waikar S. P., Lipo T.A., "Analysis and Simulation of Five-Phase Synchronous Reluctance Machines Including Third Harmonic of Airgap MMF", IEEE Transactions on Industry Applications, Vol. 3 no.2, 1998, pp 332-339.

[10] J. P. Martin, E. Semail, S. Pierfederici, A. Bouscayrol., F. MeibodyTabar, B. Davat, "Space Vector Control of 5-phase PMSM supplied by q H-bridge VSIs", ElectrIMACS'02, Montreal, August 2002, CD-ROM (common paper of GREEN and L2EP, according to the MMS project of GDR-SDSE).

[11] X. Kestelyn, E. Semail, JP. Hautier, "Vectorial Multi-machine modeling for a five-phase machine", International Congress on Electrical Machines (ICEM'O2), August 2002, Brugges (Belgium), CD-ROM.

[12] Y. Zhao Y., T. A. Lipo, " Space Vector PWM Control of Dual Three-Phase Induction Machine Using Space Vector 
Decomposition ", IEEE Transactions on Industry Applications, vol. 31 no.5, September/October 1995, pp. 1100-1109.

[13] H. Xu, H. A. Toliyat, L. J. Petersen, "Five-Phase Induction Motor Drives With DSP-Based Control System", IEEE Transactions on Power Electronics, vol. 17 no 4, July 2002, pp. 524-533.

[14] Benkhoris M.F., Terrien F., Boucher J.E. "Numerical Study of a Double Star Synchronous Motor Drive For Electrical Propulsion", International Conference on Electric Ship, Istanbul (Turkey), September 1998, pp. 70-75.

[15] G. Pasqualini, «Alimentation par convertisseurs statiques : régimes transitoires» Techniques de l'Ingénieur, traité de Génie Électrique. D3562 Volume D6., 1997.

[16] E. Semail, «Outils et méthodologie d'étude des systèmes électriques polyphasés. Généralisation de la méthode des vecteurs d'espace », Thèse de doctorat, Université des Sciences et Technologies de Lille (USTL), juin 2000.

[17] S. Gataric, "A polyphase cartesian Vector Approach To control of Polyphase AC Machines", Proc. of IEEE-IAS'O0, Rome, October 2000, CD-ROM.

[18] D. Hadiouche, "Contribution à l'étude de la machine asynchrone double étoile : modélisation, alimentation et structure", thèse de doctorat de l'UHP, Nancy 1, décembre 2001.

[19] E. Semail, A. Bouscayrol, J.P. Hautier, "Vectorial formalism for analysis and design of polyphase synchronous machines", EPJ AP (European Physical Journal-Applied Physics), vol. 22 no 3, June 2003, pp. 207-220.

[20] H.A. Toliyat, T.A. Lipo, J. C. White, “ Analysis of a Concentrated Winding Induction Machine for Adjustable Speed Drive Application Part 1 (Motor Analysis)", IEEE Transactions on Energy Conversion, Vol. 6, no.4, 1991, pp. 679-683.

[21] H.A. Toliyat, T.A. Lipo, J. C. White, « Analysis of a Concentrated Winding Induction Machine for Adjustable Speed Drive Application Part 2 (Motor design and Performance)», IEEE Transactions on Energy Conversion, vol 6 n²4, pp. 684-692, 1991.

[22] C. Hodge, S. Williamson, S. Smith, «Direct Drive Marine Propulsion Motors », International Congress on Electrical Machines (ICEM'O2), August 2002, Brugges (Belgium), CD-ROM.

[23] R. Lyra., T. Lipo, "Torque Density Improvement in a Six-Phase Induction Motor With Third Harmonic Current Injection", IEEEIAS'01, Chicago, September 2001, CD-ROM.

[24] C. Coates, D. Platt, V. Gosbell, "Performance Evaluation of a NinePhase Synchronous Reluctance Drives", Proc. of IEEE-IAS'01, Chicago, September 2001, CD-ROM.

[25] H. A. Toliyat, S. Ruhe, X. Huansheng, "A DSP-Based vector Control of Five-Phase Synchronous Reluctance Motor", IEEEIAS'OO, Rome, October 2000, CD-ROM.

[26] G. Simões, P. Vieira, "A High-Torque Low-Speed Multiphase Brushless Machine - A Perspective Application", IEEE Transactions on Industrial Electronics, vol. 49, no. 5, October 2002, pp. 1154-1164.

[27] A. Bouscayrol, Ph. Delarue, E. Semail, J. P. Hautier, J. N. Verhille, "Application de la macro-modélisation à la représentation énergétique d'un système de traction multimachine", Revue Internationale de Génie Electrique, vol. 5 n³-4, octobre 2002 pp 431-453.

[28] C. Coates, D. Platt, V. Gosbell, "Performance Evaluation of a NinePhase Synchronous Reluctance Drives", Proc. of IEEE-IAS'01, Chicago, September 2001, CD-ROM.

[29] R. Lyra., T. Lipo, "Torque Density Improvement in a Six-Phase Induction Motor With Third Harmonic Current Injection", IEEEIAS'01, Chicago, September 2001.

[30] E. Robert-Dehault, M. F. Benkhoris, E. Semail, "Study of a 5-phase synchronous machine fed by PWM inverters under fault conditions", ICEM'02, Bruges, August 2002, CD-ROM (common paper of GE44 and L2EP, according to the MMS project of GDRSDSE)

[31] J.P. Martin,"Contribution à l'alimentation en tension de machines synchrones à aimants permanents à nombre de phases elevé : fonctionnement normal et dégradé.", Thèse de Doctorat INPL Génie Électrique - E.N.S.E.M., Juillet 2003

[32] E. Semail, X. Kestelyn, "Modélisation multimachine d'une machine synchrone polyphasée ", EF'2001, Nancy, 2001, pp. 203-208.

[33] X. Kestelyn, «Modélisation vectorielle Multimachine pour la commande des ensembles convertisseurs-machines polyphasés» Thèse de doctorat, Université des Sciences et Technologies de Lille (USTL), décembre 2003.
[34] (MMS project of GdR SDSE) A. Bouscayrol, B. Davat, B. de Fornel, B. François, J. P. Hautier, F. Meibody-Tabar, M. PietrzakDavid, "Multimachine Multiconverter System: application for electromechanical drives", European Physics Journal - Applied Physics, vol. 10, no. 2, May 2000, pp-131-147 (common paper of GREEN Nancy, L2EP Lille and LEEI Toulouse).

[35] (MMS project of GdR SDSE) A. Bouscayrol, B. Davat, B. de Fornel, B. François, J. P. Hautier, F. Meibody-Tabar, M. PietrzakDavid, "Multimachine Multiconverter Systems for drives: analysis of coupling by a global modeling", Proc. of IEEE-IAS anual meeting 2000, Rome, October 2000, CD-ROM (common paper of GREEN Nancy, L2EP Lille and LEEI Toulouse).

[36] (MMS project of GdR SDSE) A. Bouscayrol, B. Davat, B. de Fornel, B. François, J. P. Hautier, F. Meibody-Tabar, E. Monmasson, M. Pietrzak-David, H. Razik, "Control structure for multimachine multiconverter systems with downstream coupling", Proc. of EPE'2001, Graz (Austria), September 2001, CD-ROM (common paper of GREEN Nancy, L2EP Lille, LEEI Toulouse and LESiR Cachan).

[37] (MMS project of GdR SDSE) A.Bouscayrol, B. Davat, B. de Fornel, B. François, J. P. Hautier, F. Meibody-Tabar, E. Monmasson, M. Pietrzak-David, E. Monmasson, H. Razik, E. Semail., M. F. Benkhoris., "Control structures for Multi-machine Multi-converter Systems with upstream coupling ", "Transactions of IMACS on Mathematical and Computers in Simulations", Vol. 63, no3-5, pp. 261-270, November 2003

[38] P. E. Vidal, M. Pietrzak-David, B. de Fornel, "Stator flux oriented control of a doubly fed induction machine", Proc. of EPE'2003, Toulouse, September 2003, CD-ROM. 